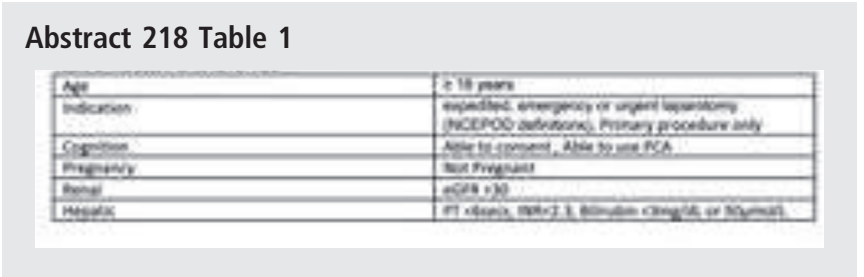

Abstract 218 Table 2

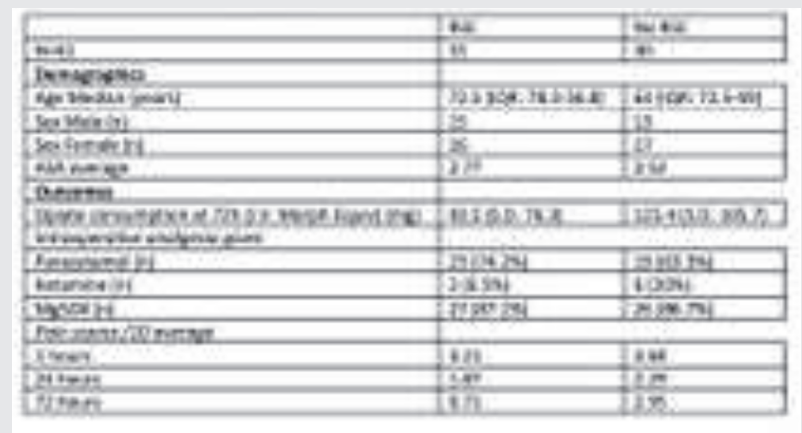

meeting local inclusion criteria for RSC at the Royal Liverpool University Hospital (table 1), September 2019 - March 2020. Results 61 patients were included. 31 (50.8\%) patients had RSC with $0.2 \%$ ropivicaine infusions running at an average $7.97 \mathrm{mls} / \mathrm{h}$ (S.D. 1.45) for a median 3 days. Median age was higher in RSC vs no RSC (72.5 vs 63 years). Patients with no RSC received alternative analgesic techniques including; spinal anaesthesia (1), transverse abdominis plane blocks (13) and IV lidocaine infusion (4). Despite this we noted a lower opioid requirement $72 \mathrm{~h}$ post-operatively in RSC patients vs no RSC (mean $93.5 \mathrm{mg}$ (S.D. 76.3) vs $125.4 \mathrm{mg}$ (S.D. 105.7)), with lower average pain scores noted at 1,24 and 72 hours in the RSC group (table 2). Ketamine use was more frequent in the no RCS group (20\% vs 6.5\%). 59 (96.7\%) received an opioid PCA post-operatively.

Conclusions Lower opioid requirements associated with RSC is consistent with other studies including a 2019 Cochrane review. The infrequent use of spinal techniques (1.6\%) may reflect concerns regarding safety in the emergency setting. Our results support available evidence, and suggest RSCs should be considered in emergency laparotomy

\section{THE CHALLENGE OF OPIOID-FREE ANESTHESIA IN NEUROSURGERY: AN OBSERVATIONAL STUDY}

R López Herrero*, B Sánchez Quirós, D Velasco Villanueva. Hospital Clínico Universitario, Valladolid, Spain

\subsection{6/rapm-2021-ESRA.219}

Background and Aims The use of opioids in the management of acute perioperative pain is very effective, but their use entails a series of adverse effects. Using OFA we avoid adverse effects. There are different studies (1) that indicate that the intra and postoperative use of opioids in patients with a tumor process could contribute to tumor progression.

Methods A retrospective observational study of patients over 18 years of age who were scheduled for elective craniectomy for a period of six months. This study was approved by the

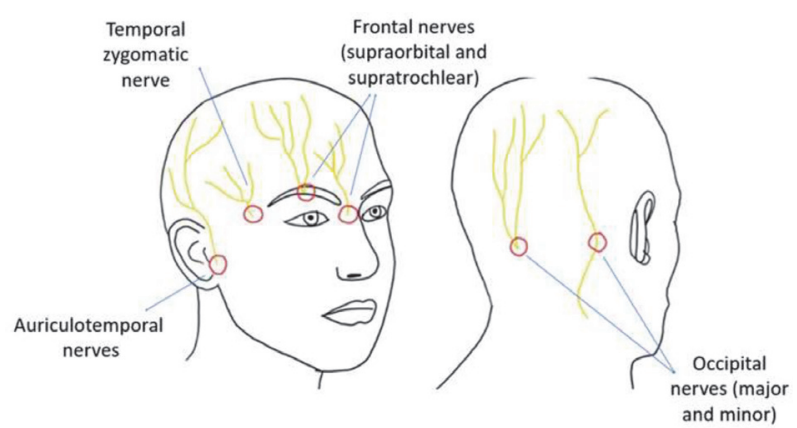

Abstract 219 Figure 1

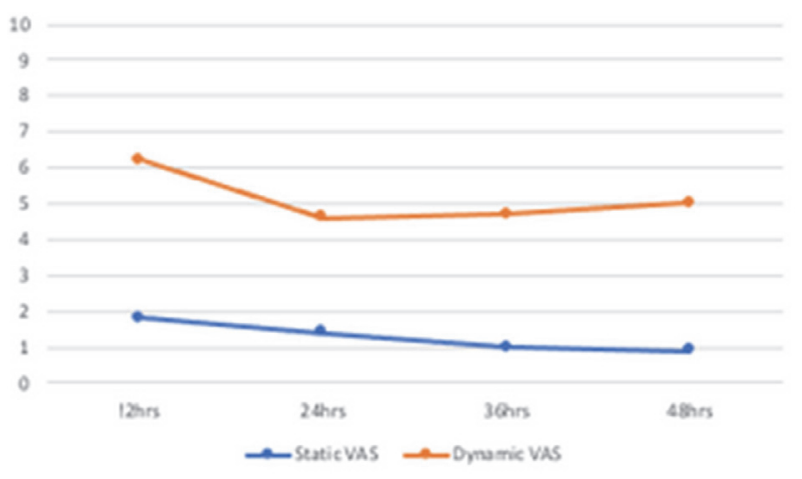

Abstract 219 Figure 2

local Ethics Committee (IP 20-1802). Anesthesia was induced with propofol, midazolam, and rocuronium. Maintenance was established with propofol. We make a scalp block (frontal, auriculotemporal, zygomaticotemporal, occipital nerves) with $0,3 \%$ ropivacaine and $1 \%$ lidocaine, using $2,5 \mathrm{ml}$ for each of the nerves. VAS values were recorded at the end of the intervention after waking up the patient and morphine doses required during the first 24 postoperative hours.

Results 46 patients were recruited, 58,7\% men and 41,3\% women, mean age $58,65 \% .76 \%$ of the patients underwent supratentorial craniectomy and 24\% underwent infratentorial craniectomy. VAS 0 after extubation in $100 \%$ of patients. $75 \%$ of the patients didn't require postoperative morphine. $26 \%$ required postoperative morphine (mean dose of $4,1 \mathrm{mg}$ in 24 hours). No patient presented complications secondary to the block.

Conclusions Although our study has limitations, there were no complications after the scalp block. We can conclude that due to the low rate of complications and the low postoperative consumption of opioids, we can use scalp block for neurosurgical.

\section{COMPARISON OF PATIENT CONTROLLED VERSUS CONTINUOUS EPIDURAL ANALGESIA IN ADULT SURGICAL PATIENTS: A SYSTEMATIC REVIEW}

${ }^{1} \mathrm{G}$ van Samkar*, ${ }^{2} \mathrm{Y} R$ Tan, ${ }^{1} \mathrm{H}$ Hermanns, ${ }^{1} \mathrm{~B}$ Preckel, ${ }^{3} \mathrm{~F}$ van Etten, ${ }^{1} \mathrm{M}$ Hollmann, ${ }^{1} \mathrm{M}$ Stevens. 'Amsterdam UMC, Location AMC, University of Amsterdam, Dept. of Anesthesiology, Amsterdam, Netherlands; ${ }^{2}$ Singapore General Hospital, Dept of Anesthesiology, Singapore, Singapore; ${ }^{3}$ Amsterdam UMC, Location AMC, University of Amsterdam, Medical Library AMC, Amsterdam, Netherlands

10.1136/rapm-2021-ESRA.220 
Background and Aims Advantages of PCEA over CEA have been demonstrated in obstetric patients. Whether similar benefit applies to surgical patients is unclear.

Aim: To assess possible advantages of patient controlled epidural analgesia (PCEA) over continuous epidural analgesia (CEA) in surgical patients

Methods Embase, PubMed and Cochrane library were searched, enabling systematic review of studies comparing PCEA and CEA in adult surgical patients (PROSPERO: CRD42018106644). Study quality was assessed using Cochrane Risk-of-Bias tool (RoB2). Primary outcome: pain score on postoperative day one (POD1). Secondary outcomes: 24 or 48 hour epidural or intravenous total analgesic dose, manual top-ups and patient satisfaction

Results Eleven trials (ten RCTs, one cohort-analysis, 1687 patients) with high heterogeneity of study characteristics were identified with a high to intermediate risk of bias. Three studies showed reduced pain scores on POD1 in PCEA compared to CEA patients $(36-42 \%, \mathrm{P}<0.05)$. Seven studies found comparable pain scores between groups, one study a higher pain score in PCEA patients. PCEA-use reduced epidural medication $(28 \%$ to $76 \%$ reduction, $\mathrm{P}<0.01)$ in seven studies. Two studies found lower top-up frequency and higher analgesic satisfaction in PCEA; PCEA patients used less intravenous morphine $(0.16$ vs $3.45 \mathrm{mg}$ per patient, $\mathrm{P}<0.05$ ) in one study.

Conclusions Regarding pain scores, rescue systemic analgesics and patient satisfaction, PCEA in surgical patients had limited advantages over CEA. PCEA reduced the amount of epidural medication and top-up frequency. On the basis of current available evidence, we cannot conclude that PCEA offers major benefits over CEA in surgical patients.

\section{IMPACT OF THE ERECTOR SPINAE PLANE BLOCK ON THE POSTOPERATIVE PAIN OF LUMBAR SPINAL STENOSIS SURGERY. A SINGLE BLIND RCT- A 70\% PATIENT'S INTERIM ANALYSIS}

${ }^{1} \mathrm{~S}$ Van Migem*, ${ }^{1} \mathrm{~N}$ Parisi, ${ }^{1} \mathrm{~L}$ Van Der Essen, ${ }^{1} \mathrm{~V}$ Marneffe, ${ }^{2} \mathrm{H}-\mathrm{M}$ Dehbi, ${ }^{1} \mathrm{G}$ Samouri. ${ }^{1}$ Clinique Saint-Pierre Ottignies, Ottignies-Louvain-la-Neuve, Belgium; ${ }^{2}$ UCL, London, UK

\subsection{6/rapm-2021-ESRA.221}

Background and Aims The erector spinae plane (ESP) block was described in 2016 by Forero et al. It involves the injection of local anesthetic into the interfascial plane, deep to erector spinae muscle, allowing the blockade of the dorsal and ventral rami of the thoracic spinal nerves. It was initially proposed for analgesia of costal fractures, pulmonary lobectomy and thoracic vertebrae. The ESP block (ESPB) could probably be extended to a large number of surgical procedures.

Methods After ethical commitee approval and informed consent, 80 patients were included in this prospective, single blind, monocentric RCT for lombar spinal stenosis surgery (LSSS) under general anesthesia: 40 patients with ESPB, 40 patients with local infiltration (LI) by the surgeon. The current interim reporting is based on 28 patients in ESPB and 28 in LI. Piritramide consumption was followed. The ESPB was realized on T12 and ultrasound-guided (chirocaine $0.25 \%+$ epinephrine 1:200.000 $4 \mathrm{mg} / \mathrm{kg}$ ). The control group was injected at the same concentration by the surgeon. Complementary analgesia was realized with Patient Controlled Analgesia (Piritramide), paracetamol and ketorolac.
Results After performing a T-test to compare the means of piritramide consumption at day 1 , we did not find any significant difference between the 2 groups (ESPB $12.9 \mathrm{mg}$ versus LI $14.7 \mathrm{mg}, \mathrm{p}=0.55)$. A Mann-Withney U-test was also performed and did not show any difference.

Conclusions After collecting data from $70 \%$ of the population, we cannot conclude that there is a benefit of ESPB over LI by the surgeon.

\section{THE USE OF REGIONAL ANAESTHESIA AND IDENTIFYING COMPARTMENT SYNDROME: A CASE REPORT}

C Hopkins*, C Broe, B Bellew. Imperial College Healthcare NHS Trust, London, UK

\subsection{6/rapm-2021-ESRA.222}

Background and Aims Acute compartment syndrome is a surgical emergency, and must be identified promptly in order to limit potential complications. The use of regional anaesthesia remains controversial for patients who are at higher risk of developing compartment syndrome due to concerns that the cardinal symptoms may be masked, and diagnosis subsequently delayed. This is a case report of a clinical situation which opposes this theory.

Methods A 31 year old male presented to a major trauma centre following a road traffic accident. Radiological imaging showed he had sustained multiple injuries, including a closed right tibia and fibula fracture and an extensive left calf laceration which required operative management. A lumbar epidural was inserted for intra-operative and postoperative analgesia, prior to the patient undergoing IM nail fixation of the right tibial fracture and exploration of the left leg laceration under general anaesthesia.

Results The lumbar epidural provided good analgesia which was maintained with a continuous infusion of $0.125 \%$ levobupivacaine and $2 \mathrm{mcg} / \mathrm{ml}$ fentanyl. Fourteen hours postoperatively, this patient developed severe right lower limb pain. Intra-compartmental pressures were measured at this stage, where it was noted that anterior compartment pressures were abnormal and the patient underwent urgent fasciotomy under general anaesthesia. Operative findings at this time were consistent with a diagnosis of compartment syndrome, with maintained viable muscle tissue present on examination.

Conclusions This case demonstrates support for alternative theories that regional anaesthetic techniques using low concentrations may provide effective analgesia without the risk of masking the presentation of acute compartment syndrome.

\section{POST OPERATIVE PAIN MANAGEMENT FOR HIP FRACTURE SURGERY, A NEW PROTOCOL EVALUATION}

${ }^{1,2} \mathrm{H} \mathrm{Ahmed}^{*},{ }^{3} \mathrm{G}$ Deloughry, ${ }^{3} \mathrm{~K}$ Joyce, ${ }^{3} \mathrm{~B} \mathrm{O}^{\prime}$ Donnell, ${ }^{3} \mathrm{~F}$ Loughnane. ${ }^{1}$ Faculty of Medicine, Cairo University, Cairo, Egypt; ${ }^{2}$ Leeds Teaching Hospitals, Leeds, UK; ${ }^{3}$ Cork University Hospital, Cork, Ireland

\subsection{6/rapm-2021-ESRA.223}

Background and Aims Hip fracture surgeries represent a significant burden on the healthcare system. Delirium could affect up to $61 \%$ of patients with hip fracture, and is associated with delayed recovery, higher morbidity and poor cognitive function. $^{(1)}$ 